\title{
DOCUMENTO
}

\section{“Fe en Europa” (Crónica desde Bruselas, marzo de 2009)'}

\author{
John Nurser ${ }^{2}$
}

"Fe en Europa" (FiE), un organismo ecuménico asociado con CTBI (Iglesias Reunidas de Reino Unido e Irlanda), organizó una visita en grupo a instituciones de la Unión Europea y parroquias asociadas viajando en el Eurostar (del 24 al 26 de febrero). Nuestro anfitrión, Matthew Ross de la Iglesia de Escocia, es miembro de lo que hasta 1999 fue la Comisión Ecuménica Europea para la Iglesia y la Sociedad (EECCS) ahora llamada Comisión de la Iglesia y Sociedad (CSC) en Bruselas (y también en Estrasburgo). La CSC es una de las comisiones de la Conferencia de Iglesias Europeas (CEC), cuya central esta en el Consejo Mundial de Iglesias (WCC) en Ginebra. Doce personas viajaron, conducidas por el presidente de "Fe en Europa" (David Thomas) y el secretario (Philip Walters), quienes junto a dos de nuestras socias, destacaban por su relativa juventud. La mayoría nacimos en los años 20 , y conservamos vívidos recuerdos de la época que iba desde Edgard Heath a Jacques Delors. Frases olvidadas como " la capellanía de Estrasburgo para las Instituciones Europeas", la "Asociación Europea de Centros Laicos", "la Cristiandad y el Futuro de Europa" (CAFÉ), "la secretaría del Consejo Británico de Iglesias Protestantes para Asuntos Internacionales", y "Teología Rural", volvieron a nuestra memoria a causa de la maraña de acrónimos y edificios antes conocidos. Éste es un informe escrito en primera persona y por tanto parcial, en ambos sentidos.

No había vuelto a Bruselas desde que me jubilé en el año 97 y por lo tanto podía comparar aquellos años con los de ahora. Nos habían dicho que el ambiente en torno a lo religioso había cambiado considerablemente con la adhesión de los

\footnotetext{
' Documento original en inglés. Traducción: Inmaculada SánChEZ BALLESTEROS.

2 John Nurser es canónigo de la Comunión anglicana en Reino Unido.
} 
países del este y que ahora había una mejor disposición para valorar la importancia de las comunidades religiosas en la variada Europa. El artículo que establece "un diálogo abierto, transparente y continuo" entre la Presidencia, las iglesias y los grupos religiosos en el propuesto Tratado de Lisboa (más sólido y específico que su preámbulo) es la evidencia de un cambio potencialmente extraordinario. Por otro lado, yo por lo menos no podía evitar un sentimiento de pérdida por la complicada desaparición del programa "Alma por Europa" de la Comisión Europea (y el Parlamento), y las víctimas que aquel asunto exigió a los posteriores socios del personal de la Comisión Ecuménica Europea para la Iglesia y Sociedad (EECCS). Aquella visión fue más compleja y mutuamente integradora. Pero tal vez estaba abocado a chocar con la tradición del laicismo anticlerical (especialmente anticatólico) de la política continental (sonaba raro a los oídos ingleses que este grupo continúe viendo la religión como una amenaza permanente a la "libertad individual".

Podría resumir mi encuentro (como sacerdote anglicano) con Europa desde 1975 básicamente como una experiencia de "visión". En Inglaterra algunos la tuvieron, la mayoría la ignoraron. Otras naciones fueron más proclives a tenerla. Aquellos en Inglaterra que la tuvieron de una forma organizada fueron los cuáqueros, los jesuitas, las ciudades hermanadas, y aquellos implicados en diversas entidades asociativas religiosas como pastorales en centros laborales, los centros laicos y los "kirchentag" (encuentros que impulsan el ecumenismo). Desde 1997 hemos sufrido un lavado de cerebro por parte de los medios de comunicación para que olvidemos los muchos políticos, empresarios y académicos que se han comprometido personalmente con un futuro ligado a Europa. Uno de ellos fue el Arzobispo, Robert Runcie.

El desorden actual del capitalismo "Anglosajón" bien podría llevar a una mayoría de Gran Bretaña a volver la vista a Europa con total convicción (sobre todo instrumental); aunque un nuevo gobierno conservador tendría que digerir primero toda una década de propaganda. Lo que sorprende es la inconsistencia de la "visión" ahora. Aquellos con los que nos reunimos en Bruselas nos confirmaron casi la total ausencia de contacto de las parroquias e iglesias británicas. Nosotros éramos el primer grupo del Reino Unido en visitar la CSC de Bruselas en 18 meses. Nuestro grupo diocesano "contact-person for Europe" informó de la absoluta apatía de la gente joven frente a su ferviente interés en el calentamiento global. Este hecho ha sido sin duda utilizado por muchos en Bruselas, incluyendo los órganos eclesiásticos con los que nos reunimos y refleja que los contactos a través de temas concretos son más efectivos que "lo visionario". 
Otro punto "estratégico" que me volvió a sorprender fue lo importante que es mirar hacia las fronteras. La posterior fusión de CEC tiene dos matrices: EECCS y la original CEC. EECCS fue en su origen una asociación laica en Bruselas de funcionarios de la Comunidad de los "Seis" (con Mark Lenders como pastor protestante de la zona a media jornada) a principios de los años 60. La OCIPE fue en su origen a una parecida asociación laica de coordinadores residentes del Consejo de Europa creada por el Obispo de Estrasburgo en la década de los 50. Por otro lado, CEC, creada para mantener el contacto con las iglesias protestantes (concretamente la EKD, Iglesia Evangélica Alemana) dividida por la intensificación del Telón de Acero; celebra este año su medio siglo teniendo su momento más decisivo en su breve historia en los acuerdos de derechos humanos de Helsinki. En aquella historia habría sido una negación radical del pasado no prestar al menos la misma atención a los estados no pertenecientes a la UE pero cuyas iglesias eran miembros de CEC, incluyendo a Noruega y las parroquias de Vladivostok. CSC está también comprometida con el Consejo de Europa en Estrasburgo, con su Tribunal de Derechos Humanos y los socios que se extienden a Turbia y Asia Central. El Festival de Eurovisión y La Copa de Europa (que son básicamente acontecimientos donde la "visión de Europa" se encuentra con el votante británico) incluyen de forma curiosa a Israel. El principal campo de acción de la OTAN es Afganistán. Nos enteramos que Chipre, (un miembro de la UE) está dentro del territorio de la Iglesia Católica Romana de Jerusalén. Y lo más sorprendente de todo, si la UE alguna vez se propusiera elegir un Presidente no cabe duda que Barack Obama ganaría sin esfuerzo.

Iniciamos nuestro viaje. Nuestro primer día fue con CSC y su organización socio católica romana COMECE (Comisión de los Episcopados de la Comunidad europea, su socia desde las Asambleas Ecuménicas Europeas desde Basilea 1989 en adelante). Dada su historia, no hay ninguna duda de la importancia de los derechos humanos en el trabajo de CEC, aunque me pregunto si es suficiente trabajar con los derechos humanos del Convenio Europeo en lugar de con aquellos recogidos en la Declaración Universal (1948). Nos contaron historias impresionantes sobre el trabajo de CSC en cuanto a los derechos humanos, el desarrollo, el diálogo interreligioso, y la integración europea. Matthew Ross comentó los próximos cambios en los fundamentos con la distribución de los beneficios de la Política Agrícola Común en el nuevo quinquenio (2013); el actual 50\% de Francia será, no sin razón, enérgicamente refutado por Polonia y otros.

COMECE, organización creada en 1980 por la Conferencia Nacional de Obispos Europeos católicos, ha cambiado y ampliado su oficina. El padre Noel Treanor, su primer secretario, se convirtió en el Obispo de Down (Belfast) el pasado otoño y 
ha sido recientemente sustituido por el padre Piotr Mazurkiewicz. El Vaticano ha tenido la oportunidad de clarificar el papel entre su segundo Nuncio en Bruselas (desde finales de los 80 hasta la Unión Europea), COMECE y OCIPE. La perspectiva actual de COMECE no es teológica sino sobre asuntos que tienen que ver con "el bien común" y "el estilo de vida". Cuentan con un nivel europeo (tanto nacional como diocesano) donde la acción común con CSC ha adquirido una regularidad importante por ejemplo en los temas sobre el cambio climático. Sin embargo, dos temas significativos de la Iglesia Católica Romana surgieron en la conversación: en particular una pregunta sobre un panfleto común de CSC/COMECE en asuntos sobre las iglesias locales para proponer los candidatos para la elección al Parlamento de junio de este año. Quedó claro que COMECE había rechazado los principios liberales del documento de CSC a favor de exponer requerimientos específicos de la Iglesia Católica Romana tales como el aborto y no quedó ninguna duda de que la falta de preparación de Roma (podríamos decir lo mismo de Moscú como "Tercera Roma") para aceptar un situación igualitaria con la Cienciología, por poner un ejemplo, en el plan de reuniones habituales propuestas con la Presidencia de la UE. Según nos dijeron, el 60\% de la población de la UE es "católica".

El segundo día comenzó en el nuevo edificio del Parlamento Europeo (que cuenta con una sala de meditación). Parte de su razón de ser fue el rebelde consenso por parte de los miembros del Parlamento Europeo que consideraban absurdo, costoso y un aburrimiento tener que unirse a una masiva caravana para ir y volver de Estrasburgo para sus sesiones regulares. Todo para agradar a los presidentes franceses. Así que se construyó un increíble templo de mármol donde el espacio no ha sido un problema. Pero Francia, hasta ahora, no ha cedido/cambiado de opinión. La mayoría de los miembros del Parlamento regresan a sus circunscripciones durante una semana al mes (los nuestros aquella semana, y además era Miércoles de Ceniza), por lo que estuvimos encantados de encontrar al lider del Partido Liberal Europeo, Andrew Duff, en casa para conocernos. Él había estado muy implicado en el borrador del aún no resuelto Tratado de Lisboa que expuso como una eficaz base estructural para la integración en curso de la UE. Habló efusivamente de las complejidades de la "sociedad civil" europea, de los institutos de la mujer locales, y de la legítima importancia de las iglesias. Le planteamos el alejamiento de algunos de los miembros del Parlamento de su mega-electorado pero él enfatizó que eso tenía poca importancia comparado con el hecho de lograr una representación proporcional. 2008 fue "un año de diálogo intercultural" y el comisario cultural (un eslovaco) hizo que la religión fuera un punto principal. Más cerca de casa para los miembros del aparato de la UE, nos dijeron que la población de Bruselas dentro de diez años será musulmana lo que podría acentuar la importancia de las ortodoxias actuales de "dialogo" y "pluralismo religioso". 
Parece que la Comisión ha dicho que no desea ninguna competencia en "educación", algo que un ayudante de un miembro del Parlamento Europeo considera equivocado.

Tras la reunión era obligada una visita a la Pro-Catedral Anglicana, pero yo elegí ver a un viejo amigo que trabajaba en el Parlamento Europeo (un seglar de la Iglesia Católica que ha tomado la jubilación anticipada para un encuentro profundo con el Islam), y después asistí a la muy concurrida misa del Miércoles de Ceniza en la nueva Capilla de la Resurrección (un antiguo convento a medio camino del Parlamento y la Comisión adaptado para uso del personal de la UE).

La Casa Quaker ("Quaker House") es, sin lugar a dudas, el éxito de la historia del "acompañamiento" cristiano en el desarrollo de "Europa" en Bruselas. La Sociedad de Amigos cuenta, después de todo, con muy buenas citas de William Penn desde hace ya más de 300 años. Su gran casa familiar ex-burguesa abrió sus puertas con una pareja inglesa, los Stapletons, como guardianes en 1979, tras la esmerada formación de un Consejo Cuáquero transnacional de asuntos europeos. La reflexión anterior al establecimiento en 1984 de un representante anglicano para las Instituciones europeas en Estrasburgo debió mucho a este concepto pionero. Los guardianes (un antiguo guardián iba en nuestro grupo) tienen su hogar en la Casa, junto con dos o tres jóvenes en prácticas por lo que hay una comunidad que ofrece hospitalidad y un centro para el culto confesional habitual. Hay una sala de reuniones. Se ha creado una biblioteca y se publican trabajos y casi de forma única hay un verdadero ir y venir de amigos de los estados miembros. No sorprende que la Oficina Europea de Enlace de Consolidación de la Paz y la Sociedad Civil del Grupo de Contacto son en gran parte logros cuáqueros. Se nos sugirió que un motivo para la indudablemente "mísera subfinanciación" del Consejo de Europa es la intención de los Estados de retrasar las apelaciones a la Corte de los Derechos Humanos.

La tarde terminó con una visita a la innovación estructural de Jean Monnet, la Comisión Europea (en el renovado Berlaymont) que ha transformado el arte de gobernar europeo de los últimos 50 años. No era buen día para visitas. El Reino Unido (como todos los otros 27 estados miembros) tiene ahora un comisario, en este caso Lady Ashton Comisaria de Comercio Exterior que sucedió a Peter Mandelson. La actual crisis económica/financiera implica que haya actualmente preparativos urgentes en sus oficinas organizando algunas reuniones internacionales muy importantes. Cada comisario tiene un "gabinete" de personas designadas y una de esas (con experiencia en Asuntos Exteriores) habló con nosotros muy brevemente. Se tomó la molestia de asegurarnos que el objetivo de la Comisión es siempre " ¿lo mejor 
para Europa?" y entonces, "¿cómo puede encomendarse a los estados miembros?". Aparentemente es "automático" incluir la religión al usar la frase "sociedad civil". Nos sorprendió una señal que vimos al salir del ascensor; ésta era un "Colegio" de comisarios bajo un presidente-una tradición muy benedictina-. Se reunen "en sede" todos los miércoles.

Nuestro último día empezó con una visita a OCIPE, la Oficina Jesuita Europea, fundada por el Arzobispo de Estrasburgo a petición de los seglares del Consejo de Europa en 1956 y que trasladó su oficina principal a Bruselas en 1963. Es, por consiguiente, la institución católica más antigua creada (con un verbo revelador) "para acompañar la construcción de Europa"; con un fuerte compromiso a la "idea" europea. Su trabajo entonces estaba en las relaciones personales con los funcionarios de la UE, la publicación de artículos, el pensamiento crítico, y la información a través de las provincias de la Compañía de Jesús en Europa. Aquí de nuevo nos encontramos con una bastante modesta oficina en un nuevo emplazamiento. El padre Frank Turner, el director, es inglés y ha permanecido en el puesto desde 2005. Hay oficinas filiales en Varsovia y Budapest y aún una presencia parcial en Estrasburgo. El énfasis actual está en el apoyo activo internacional a la investigación, con especial interés en la justicia en la explotación de recursos minerales por las empresas transnacionales (ETN) en el Congo y por los "dálits" (o intocables) en la India. Un asunto de vital importancia (que incluye a algunos musulmanes) sigue siendo la energía y el cambio climático.

Antes de salir para el Eurostar nos vimos de nuevo con Matthew Ross en CSC para repasar nuestras experiencias juntos y planear el próximo acontecimiento del FiE en el otoño (en Dunblane).

Desde mi punto de vista hay cuatro asuntos que demandan una atención especial y urgente de las iglesias británicas:

1. Si la enorme labor puesta en el borrador del Tratado de Lisboa se cristaliza en determinar la práctica de la UE (quizás demasiado pronto si un segundo voto irlandés fuese "si"), entonces su artículo 17 se convertiría en un requerimiento legal de la UE: "Reconociendo su identidad y su contribución particular, la Unión mantendrá un diálogo abierto, transparente y regular con las asociaciones o comunidades eclesiásticas y religiosas". Esta obligación ha sido también aceptada por el Consejo de Europa. Es difícil enfatizar sobradamente el significado a largo plazo de este momento, o la importancia de obtener los mecanismos (por tanto los precedentes) justo desde el principio. No está claro si este diálogo está previsto en una serie de reuniones (¿¿de diferentes comisiones?) con grupos 
religiosos específicos (católico romanos, humanistas, musulmanes, pentecostales, etc), o si un debate general que incluya a todos está en mente (lo que podría no ser útil). ¿ Quién decide la orden del día? y ¿̇a quíen se le entregaran las actas? La Iglesia de Inglaterra tiene una posición distinta, por un lado en lo teológico y por otro en estar circunscrita a sólo un estado miembro, y ahora en tener a un representante elegido para la UE. CEC tiene una honorable tradición en el papel pero no es indisputablemente apropiada como instrumento común, en ambos casos por su limitada experiencia y por su inclusión en la muy amplia iglesia ortodoxa de Rusia (que no es un estado miembro).

2. ¿Cuál es la intención de las elecciones "democráticas" al Parlamento Europeo, y hay alguna razón en que CSC se confabule (a cualquier precio) con el actual choque entre la retórica y la realidad? La idea de un foro transnacional para que políticos nacionales elegidos encuentren compañeros es evidentemente útil- como en el Consejo de Europa. La retórica de un Parlamento de la UE ligeramente "confederal" requiere una clara diferenciación como la que hay por ejemplo en las elecciones australianas entre el Estado y la Commonwealth dentro de la organizaciones de partido, plataformas electorales y liderazgo. La realidad actual (al menos en Inglaterra) es que los partidos en Westminster están decididos a conservar el control de la participación en el Parlamento Europeo; de manera que no haya ningún interés popular, ningún electorado mínimo con relación a los miembros del Parlamento Europeo, ni conciencia de quienes son las figuras de liderazgo en los partidos del Parlamento Europeo, ni atención de los medios de comunicación, y ningún conocimiento de los "asuntos" a decidir en cada voto emitido.

3. Con respecto al trabajo de la Comisión de Iglesia y Sociedad (CSC) de la Conferencia de Iglesias Europeas (CEC) y a su admirable, trabajador (y reducido) personal, varios nos preguntamos si los comisarios deberían discutir como podría su trabajo ser menos agotador y más rentable. Está claro que hay una necesidad fundamental de que las iglesias tengan acceso y confianza en las "personas" de CSC que saben moverse entre los acrónimos y los funcionarios de las instituciones de Bruselas, y quienes pueden, cuando se les pide, ser defensores de una cierta política. La actualización periódica de la CSC en Asuntos Europeos cubre una autentica necesidad y debería divulgarse entre todos los "delegados europeos" diocesanos (y equivalentes). Recibir a los grupos visitantes es algo que lleva mucho tiempo, pero importante también pues es una vinculación con otros grupos cristianos en Bruselas. Se le exige mucho tiempo al personal en considerables redacciones de proyectos que deben ser fidedignos para demostrar su utilidad y es posible que no se tome el suficiente 
cuidado en establecer el tiempo necesario para estos asuntos, (ininterrumpidos por los frecuentes vuelos). Podría ser también útil sopesar la utilización de las publicaciones de CSC por parte de las iglesias constituyentes: ¿las solicitaron? ¿se esperaba que el clero les encontrase los recursos básicos? ¿llegaban a tiempo de ser utilizados? ¿̀hay información posterior?

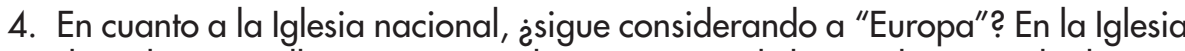
de Inglaterra se llegó a un acuerdo a principios de los 90 de que cada diócesis eligiera a su "funcionario en Europa". Tal vez haya llegado la hora para que la Cámara de Obispos ponga este asunto en el orden del día. Nuestro grupo incluía a una seglar que se encarga de la oficina de St Albans, una diócesis con una larga histora de auténtico interés en construir la conciencia de "Europa". Su homólogo (un sacerdote) en la diócesis de Manchester y con una parecida contribución a la historia, tuvo que abandonar. Hay, sin embargo, otras diócesis en las cuales (si el título aún existe), hay una insignificante actividad y ninguna forma de acceder a la información. El reciente ejemplo del Obispo Herbert en San Albans demostró la diferencia que supone una diócesis comprometida.

Y por último, una de mis obsesiones. ¿Deberíamos pensar sobre la orientación religiosa general de la sociedad que (aunque lentamente) está tomando forma en los territorios de la UE? Y arriesgándome, tengo la impresión de que mientras se considere que los participantes activos más importantes de la escena religiosa sean del "Protestantismo bíblico", "Catolicismo Vaticano", y "Tradicionalismo del Islam", ninguno de ellos parecerá estar en la dirección tomada por la religiosidad no expresada de la gran mayoría de los ciudadanos de la UE ly los hombres y mujeres que trabajan en las instituciones de la EU). Un historicamente "revelador" texto sagrado o sublimemente establecido en la alta jerarquía eclesiástica pone curiosamente la nota discordante. Mientras las teologías de los cuáqueros o de los benedictinos, o de la religiosidad oriental, no. 